\title{
LinearFold: Linear-Time Prediction of RNA Secondary Structures
}

\author{
Dezhong Deng ${ }^{\mathrm{a}}$, Kai Zhao ${ }^{\mathrm{b}, 1}$, David Hendrix ${ }^{\mathrm{c}, \mathrm{a}}$, David H. Mathews ${ }^{\mathrm{d}}$, and Liang Huang ${ }^{\mathrm{a},{ }^{*}}$ \\ ${ }^{a}$ School of Electrical Engineering and Computer Science, Oregon State University, Corvallis, OR; ${ }^{\mathrm{b}}$ Google, Inc., New York, NY; ${ }^{\mathrm{c}}$ Dept. of Biochemistry \& Biophysics, Oregon \\ State University, Corvallis, OR; ${ }^{\mathrm{d}}$ Dept. of Biochemistry \& Biophysics, Center for RNA Biology, and Department of Biostatistics \& Computational Biology, University of Rochester \\ Medical Center, Rochester, NY
}

This manuscript was compiled on February 13, 2018

Predicting the secondary structure of an RNA sequence with speed and accuracy is useful in many applications such as drug design. The state-of-the-art predictors have a fundamental limitation: they have a run time that scales cubically with the length of the input sequence, which is slow for longer RNAs and limits the use of secondary structure prediction in genome-wide applications. To address this bottleneck, we designed the first linear-time algorithm for this problem. which can be used with both thermodynamic and machine-learned scoring functions. Our algorithm, like previous work, is based on dynamic programming (DP), but with two crucial differences: (a) we incrementally process the sequence in a left-toright rather than in a bottom-up fashion, and (b) because of this incremental processing, we can further employ beam search pruning to ensure linear run time in practice (with the cost of exact search). Even though our search is approximate, surprisingly, it results in even higher overall accuracy on a diverse database of sequences with known structures. More interestingly, it leads to significantly more accurate predictions on the longest sequence families in that database (16S and 23S Ribosomal RNAs), as well as improved accuracies for long-range base pairs $(500+$ nucleotides apart).

RNA | secondary structure prediction | linear-time $\mid$ dynamic programming $\mid$ beam search

$\mathbf{R}$ ibonucleic acid (RNA) is involved in numerous cellular processes. While many RNAs encode proteins (messenger RNAs, mRNAs), noncoding RNAs (ncRNAs) have intrinsic functions without being translated to proteins (1). ncRNA sequences catalyze reactions $(2,3)$, regulate gene expression (4-6), provide site recognition for proteins $(7,8)$ and serve in trafficking of proteins $(9)$. The recent discovery and characterization of diverse classes of long noncoding RNAs, i.e. ncRNAs longer than $200 n t$ (10), present new opportunities and challenges in determining their functions and mechanisms of action. Furthermore, the dual nature of RNA as both a genetic material and functional molecule led to the RNA World hypothesis, that RNA was the first molecule of life (11), and this dual nature has also been utilized to develop in vitro methods to evolve functional sequences (12). Finally, RNA is an important drug target and agent (13-18).

Predicting the secondary structure of an RNA sequence, defined as the set of all canonical base pairs (A-U, G-C, G-U), is an important and challenging problem $(19,20)$. Knowing structures reveals crucial information about the RNA's function, which is useful in many applications ranging from ncRNA detection (21-23) to the design of oligonucleotides for knockdown of message $(24,25)$. Being able to rapidly determine the structure is useful given the overwhelming increase in genomic data (about $10^{21}$ base-pairs per year) (26) and given the small percentage of sequences that have experimentally determined structure. Experimental assays can provide information that can improve the accuracy of RNA secondary structure prediction (27), and these assays can now be used transcriptome-wide and in vivo (28-30). Recent studies focused on improved accuracy of prediction
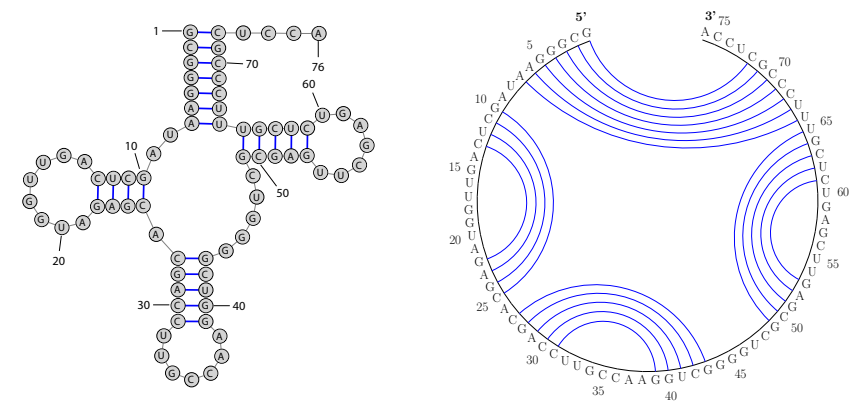

GCGGGAAUAGCUCAGUUGGUAGAGCACGACCUUGCCAAGGUCGGGGUCGCGAGUUCGAGUCUCGUUUCCCGCUCCA $4(4+1+4(4+\cdots)$

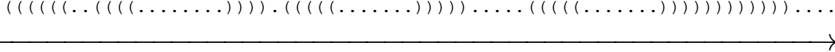

\begin{tabular}{c|cc|cc}
\hline 1 & \multicolumn{3}{c}{ our linear-time algorithm scans left-to-right } & \\
& \multicolumn{2}{|c}{ efficiency } & \multicolumn{2}{c}{ systems } \\
& time & memory & machine-learned & thermodynamic \\
\hline baseline & cubic & quadratic & CONTRAfold & Vienna RNAfold \\
our work & linear & linear & LinearFold-C & LinearFold-V
\end{tabular}

Fig. 1. RNA secondary structure and high-level idea of our work. Top left: secondary structure of E. coli tRNA ${ }^{\text {Gly }}$; Top right: the corresponding circle plot; Central: the corresponding dot-bracket format. Bottom: schematic view of our work. In a nutshell, our algorithm scans the sequence left-to-right, and tags each nucleotide as "." (unpaired), "(" (to be paired with a future nucleotide) or ") " (paired with a previous nucleotide).

\section{Significance Statement}

Fast and accurate prediction of RNA secondary structures (the set of canonical base pairs) is an important problem, because RNA structures reveal crucial information about their functions. Existing approaches can reach a reasonable accuracy for relatively short RNAs but their running time scales almost cubically with sequence length, which is too slow for longer RNAs. We develop the first linear-time algorithm for RNA secondary structure prediction. Surprisingly, our algorithm not only runs much faster, but also leads to higher overall accuracy on a diverse set of RNA sequences with known structures, where the improvement is significant for long RNA families such as $16 \mathrm{~S}$ and $23 \mathrm{~S}$ Ribosomal RNAs. More interestingly, it also more accurate for long-range base pairs.

Author contributions: L.H. conceived the idea based on D.H.'s suggestion. L.H., D.D., and K.Z. designed the algorithm. L.H. and D.D. implemented a prototype in Python. D.D. and K.Z. implemented the fast version in C++. D.H.M. and D.H. supervised testing of the algorithm. D.D. carried out testing and plotted figures. D.D., L.H., and D.H.M. wrote the manuscript; D.H. revised it.

The authors declare no conflict of interest.

${ }^{1}$ K.Z.'s contribution was done at School of EECS, Oregon State University.

To whom correspondence should be addressed. E-mail: liang.huang. shegmail.com 
bioRxiv preprint doi: https://doi.org/10.1101/263509; this version posted February 14, 2018. The copyright holder for this preprint (which was not certified by peer review) is the author/funder, who has granted bioRxiv a license to display the preprint in perpetuity. It is made available under aCC-BY-NC-ND 4.0 International license.

(31-35), but there is not enough attention on the speed of prediction.

While there are two major approaches to modeling RNA secondary structures, namely the classical thermodynamic methods $(36,37)$ and the more recent machine learning-based methods $(38,39)$, all these efforts use virtually the same dynamic programming (DP) algorithm $(40,41)$ to find the best-scoring structure. However, this algorithm, borrowed from computational linguistics $(42,43)$, has a running time of $O\left(n^{3}\right)$ that scales cubically with the sequence length $n$. This is slow for long RNAs $(n>1,000)$, and in practice, many researchers resort to running this algorithm on short regions within the whole sequence, which inevitably ignores base pairs across segments (44). Computational and experimental studies demonstrate that base pairing between the ends of natural RNA sequences is expected.

In this paper, we design the first linear-time RNA secondary structure prediction algorithm, LinearFold, inspired by our previous work on linear-time natural language parsing (45). While the classical $O\left(n^{3}\right)$ dynamic programming is bottom-up, solving the best substructure for each span, our algorithm is left-to-right, incrementally tagging each nucleotide in the dot-bracket format (unpaired ".", opening " (", or closing ")"). While this naive version runs in exponential time $O\left(3^{n}\right)$, we use an efficient merging approach borrowed from computational linguistics (46) that reduces the running time back to $O\left(n^{3}\right)$. On top of this left-to-right algorithm, we further apply beam search, a popular heuristic to prune the search space (45), which keeps only the top $b$ highest-scoring states at each nucleotide, resulting in an $O(n)$ time approximate search algorithm. Even though our search is not exact, empirically, with a reasonable beam size ( $\operatorname{such}$ as $b=100$ ) it is close to exact search, and actually leads to better prediction accuracies than exact search.

Our algorithm can be used with both thermodynamic and machine learned models. In particular, we implemented two versions of LinearFold, LinearFold- $V$ using the thermodynamic free energy model from Vienna RNAfold (37), and LinearFold-C using the machine learned model from CONTRAfold (38) (see Fig. 1 (bottom)). We evaluate our systems on a diverse dataset of RNA sequences with well-established structures, and show that while being substantially more efficient, LinearFold leads to higher average accuracies over all families, and somewhat surprisingly, LinearFold is significantly more accurate than the exact search methods on the longest families $16 \mathrm{~S}$ and 23S Ribosomal RNAs. More interestingly, LinearFold is also more accurate on long-range base pairs that are more than 500 nucleotides apart, which is well known to be a challenging problem for the current models (47).

\section{Results}

Efficiency and Scalability of LinearFold. To demonstrate the efficiency and scalability of LinearFold, we compare its running time with the conventional cubic-time prediction algorithms used in the baseline systems, CONTRAfold and Vienna RNAfold. Figure 2 shows the results on two datasets: (a) the ArchiveII dataset (48), a diverse set of RNA sequences with known structures (see details in the Methods section and Table SI 1), and (b) a (sampled) subset of RNAcentral (49), a comprehensive (meta-)set of ncRNA sequences from many databases. While the ArchiveII set contains sequences of length 3,000 or less, the RNAcentral set has many much longer sequences, with the longest being 244,296 nt (Homo Sapiens Transcript NONHSAT168677.1, from the NONCODE database (50)). We use a machine with 3.40GHz Intel Xeon CPUs and 32G memory, running Linux; all programs are written in C/C++ compiled by GCC 4.9.0.

Figure 2 A confirms that LinearFold's running time scales linearly with the sequence length, while the two baseline systems scale super-
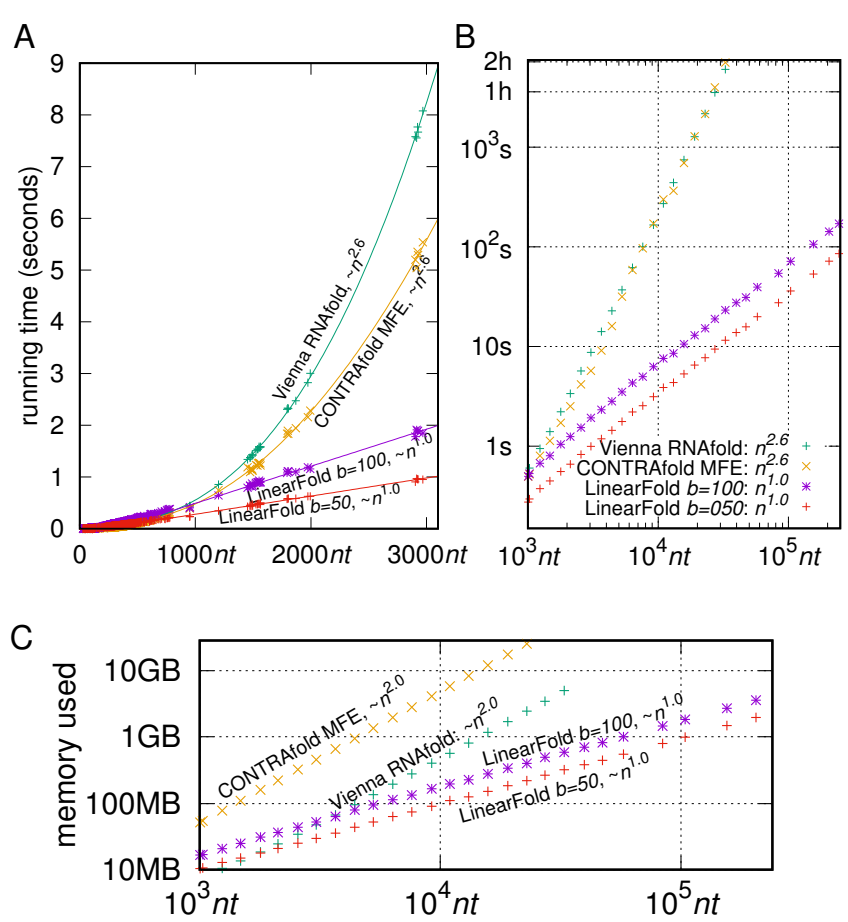

Fig. 2. A: runtime comparisons on Archivell dataset: LinearFold- $C$ (with beam sizes 100 and 50) vs. two baselines, CONTRAfold MFE \& Vienna RNAfold (LinearFold-V have identical running time with LinearFold-C). B: runtime comparisons on RNAcentral dataset (log-log). C: memory usage comparisons (RNAcentral set, log-log).

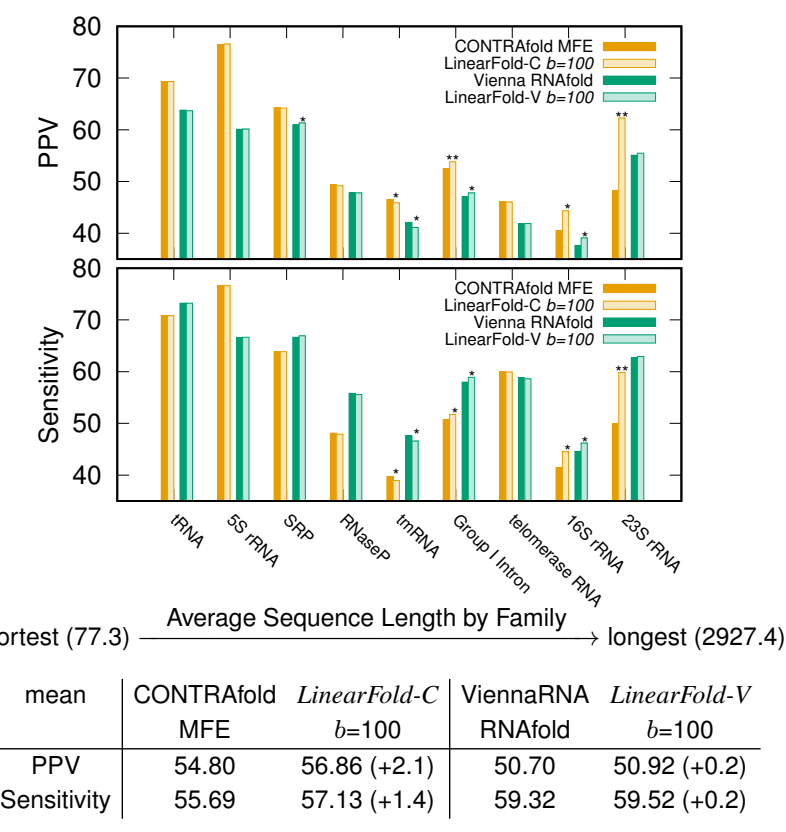

Fig. 3. PPV and Sensitivity (by family) on the Archivell dataset, comparing LinearFold with the corresponding baselines, CONTRAfold MFE and Vienna RNAfold. Each column represents a family accuracy, averaged over all sequences in that family. The overall accuracies are reported in the table, averaging over all families. Statistical significance is marked as ${ }^{*}(0.01 \leq p<0.05)$, and ${ }^{* *}(p<0.01)$. See Table SI 1 for detailed accuracy numbers. See the Methods section for details of the PPV/Sensitivity metrics and the significance testing method. 

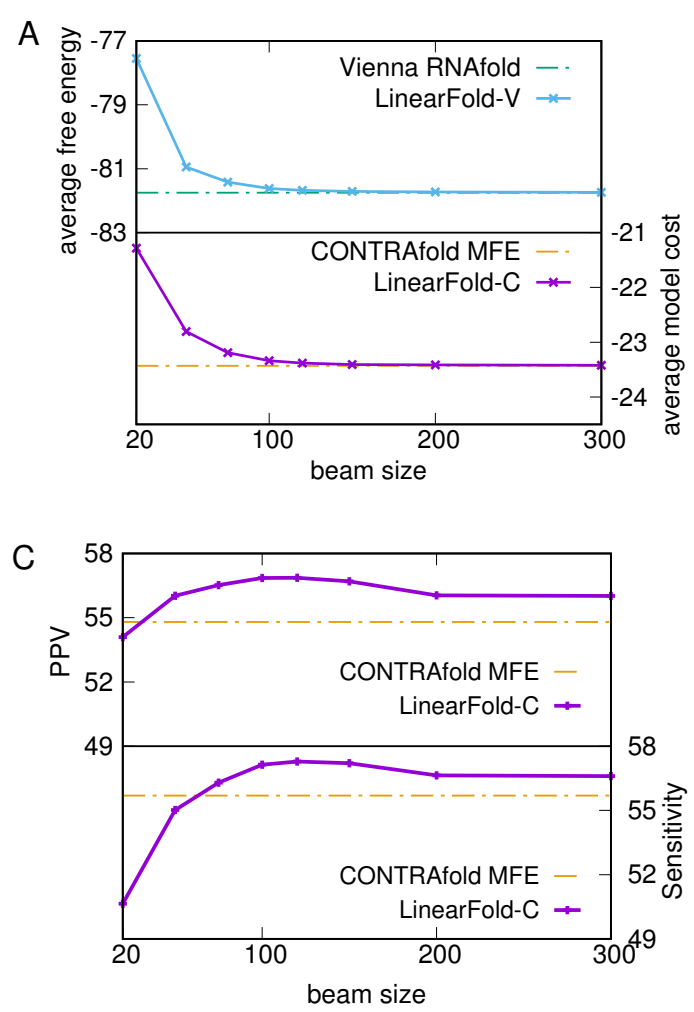
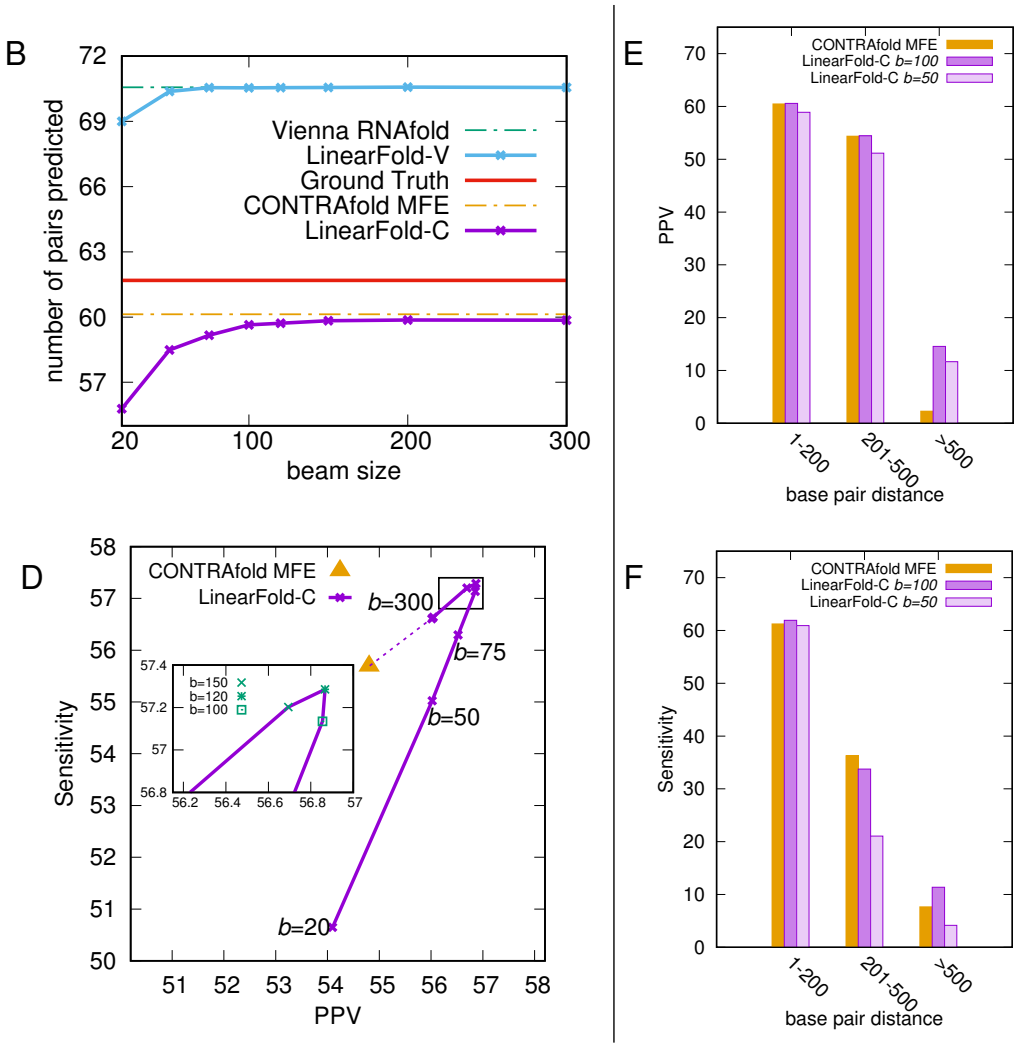

Fig. 4. Impact of beam size. A-D illustrate the trends of different variables when beam size increases. A: the internal cost, namely averaged free energy / model cost of two versions of LinearFold; B: the number of pairs predicted (averaged by sequence) of these methods, comparing with Ground Truth; C: change of both PPV and Senstivity with the increasing of beam size; D: PPV-Sensitivity tradeoff when varying beam size; E-F: PPV and Sensitivity against pair distance in the Archivell dataset, comparing LinearFold- $C$ with CONTRAfold MFE. Each point represents the overall PPV/Sensitivity of all base pairs in a certain length range.

linearly, with an empirical runtime of $O\left(n^{2.6}\right)$ determined by curve fitting. Figure $2 \mathrm{~B}$ reconfirms this fact on much longer sequences (in log-scale), and for a sequence of $\sim 10,000 n t$ (e.g., the HIV genome), LinearFold (with the default beam size of $b=100$ ) takes only 7 seconds while the baselines take 4 minutes. For sequences of length 32,753, our LinearFold takes only 23 seconds while CONTRAfold takes 2 hours and RNAfold 1.7 hours. This clearly shows the advantage of our linear-time prediction algorithm on very long ncRNAs.

In addition, LinearFold also has an advantage on memory usage that leads to better scalability on extremely long sequences. The baseline cubic-time algorithms require memory space that scales quadratically with sequence length, because intuitively they need to figure out the best scoring or minimum free energy structure for every substring $[i, j]$ of the entire sequence. This means you need $4 \mathrm{x}$ memory if your sequence length doubles. In addition, due to a design deficiency, neither CONTRAfold MFE or Vienna RNAfold runs on any sequence longer than 32,767 $n t$. On the other hand, LinearFold not only takes linear time, but also uses linear memory, without the need for the two-dimensional table of size $O\left(n^{2}\right)$. As a result, LinearFold is able to process the longest sequence in RNAcentral $(244,296 n t)$, taking less than 3 minutes. In fact, LinearFold even scales to sequences of $10,000,000 \mathrm{nt}$ on our 32GB-memory machine.

Accuracy of LinearFold. We next compare the prediction accuracies of LinearFold and the two baseline systems, reporting both Positive Predictive Value (PPV; the fraction of predicted pairs in the known structure) and Sensitivity (the fraction of known pairs predicted) on each RNA family in ArchiveII dataset. We also tested statistical signif- icance using a paired, two-tailed $t$-test, following previous work (51). Figure 3 shows that LinearFold-C improves PPV and Sensitivity over CONTRAfold by $+2.1 \% /+1.4 \%$ (absolute)when averaged across all families. This is surprising because LinearFold produces more accurate structures using a fraction of runtime. Individually, LinearFold-C is significantly more accurate in both PPV/Sensitivity on three families: Group I Intron, $16 \mathrm{~S}$ and $23 \mathrm{~S}$ ribosomal RNAs, with the last two being the longest families in this dataset. 16S rRNAs have an average length of $1548 n t$ and $+3.89 \% /+3.08 \%$ absolute improvement in PPV/Sensitivity, and 23S rRNAs have an average length of $2927 \mathrm{nt}$ and $+14.00 \% /+9.98 \%$ absolute improvement. Even more surprising, LinearFold's improvement in accuracy is more pronounced on longer sequences. Accuracies are also improved by LinearFold- $V$ over Vienna RNAfold, but the difference is smaller (overall $+0.2 \% /+0.2 \%$ absolute improvement in PPV/Sensitivity). Individually, the improvement is significant on both PPV/Sensitivity on two families, Group I Intron and 16S rRNA.

Impact of Beam Size. Above we used $b=100$ as the default beam size. Now we investigate the impact of different beam sizes. We first study the impact of search quality. Since our LinearFold algorithm uses approximate search instead of exact search, we use the difference between exact search free energy and our returned free energy as the measure of search quality - the closer they are, the better our search quality. We can see from Figure $4 \mathrm{~A}$ that the search quality is getting closer when the beam size increases, and LinearFold achieves similar model cost / free energy using the default beam size. Similarly, Figure 4 B plots the number of pairs predicted, at 
bioRxiv preprint doi: https://doi.org/10.1101/263509; this version posted February 14,2018 . The copyright holder for this preprint (which was not certified by peer review) is the author/funder, who has granted bioRxiv a license to display the preprint in perpetuity. It is made available under aCC-BY-NC-ND 4.0 International license.

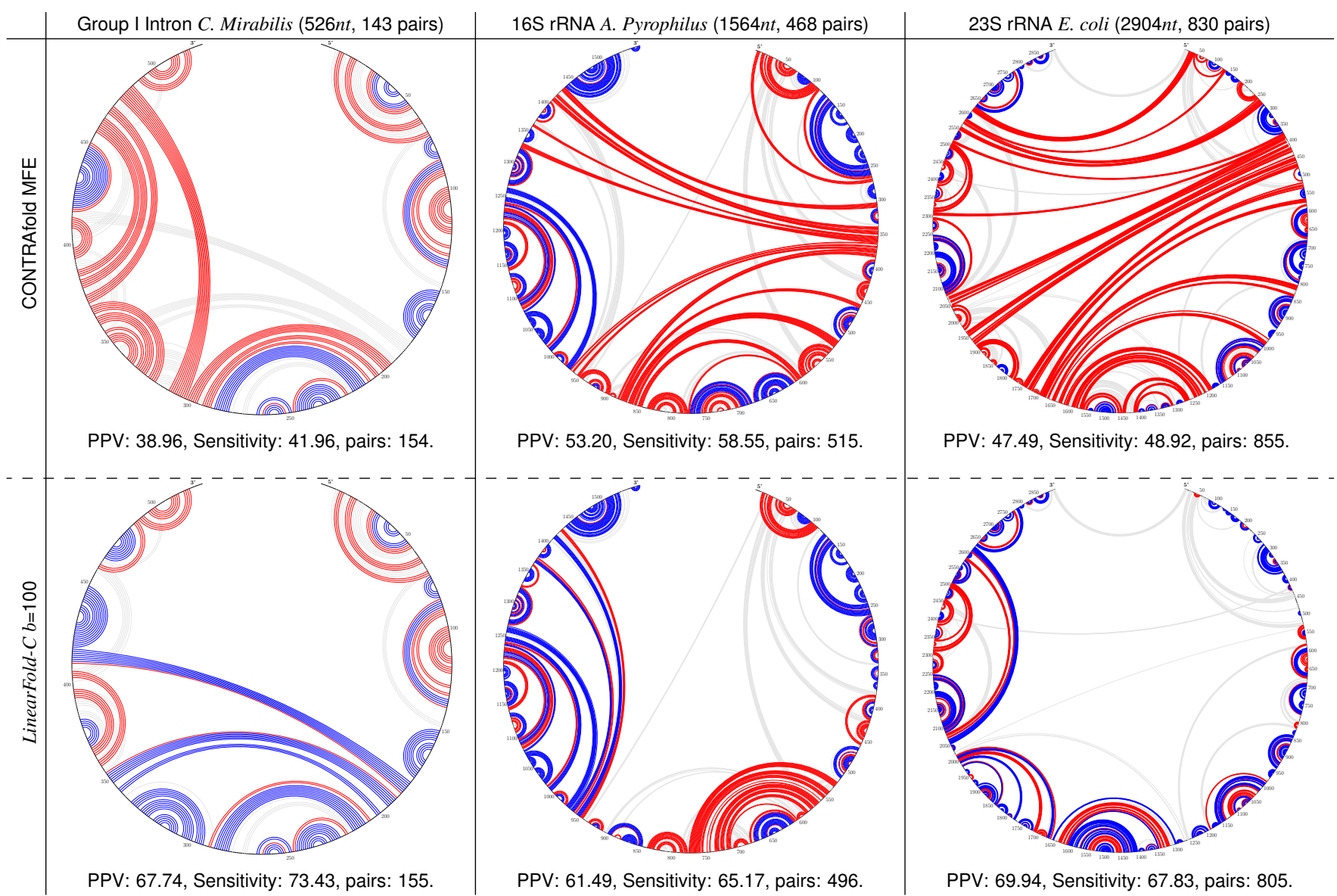

Fig. 5. Circular plots of 3 RNA sequences (selected from 3 different RNA families) comparing CONTRAfold MFE with LinearFold-C. A blue arc represents a correctly predicted pair, a red arc represents an incorrectly predicted pair, while a light gray arc represents a based pair miss predicted. Each plot uses the clockwise order of the RNA sequence from the top. These 3 examples are picked from 3 different RNA families that the performance of LinearFold-C is improved significantly comparing to CONTRAfold MFE.

each beam size. It shows that ViennaRNA model tends to overpredict, while the CONTRAfold model underpredicts. Also, although our algorithm always underpredicts compared to exact predictions in either model, we predict almost the same number of base pairs as each model when using the default beam size. The mean difference is 0.29 (CONTRAfold)/0.01(ViennaRNA) pairs when $b=100$.

Figure $4 \mathrm{C}$ plots PPV and Sensitivity as a function of beam size $b$. LinearFold-C outperforms CONTRAfold MFE in both PPV and Sensitivity with $b \geq 75$ (though it will converge to CONTRAfold MFE when $b \rightarrow+\infty)$, and LinearFold-C's PPV/Sensitivity are stable with $b \in[100,150]$. Figure 4 D shows the tradeoff of PPV and Sensitivity of LinearFold, with the change of the beam size. It starts with the increasing of both PPV and Sensitivity, reaches the peak at $b=120$, and falls until converging to exact search. Although the peak happens when beam size is 120 , we can see that the performance LinearFold is consistent when its beam size is in $[100,150]$, as both PPV/Sensitivity stays almost the same.

Accuracy Improvements on Long-Range Base Pairs. We further evaluated the effect of the distance between base pairednucleotides on prediction performance. As shown in Figure $4 \mathrm{E}$ and F, when predicting long-distance pairs, LinearFold can outperform previous approaches in both PPV and Sensitivity. Contrary to the concern that LinearFold would not predict as accurately for long-distance pairs, it continues to outperform previous methods even at pairing distances over $500 \mathrm{nt}$. Detailed comparisons between LinearFold-V and Vienna RNAfold in PPV, Sensitivity, and prediction quality, are in the Supporting Information.

Example Predictions: Group I Intron, 16S and 23S rRNAs. We visualized the predicted secondary structure of 3 examples from different RNA families, Group I Intron C. Mirabilis, 16S rRNA A. Pyrophilus, 23S rRNA E. coli, comparing LinearFold-C with CONTRAfold MFE (Figure 5). The circular plots show our performance improvement, by both predicting more base pairs correctly, and reducing incorrect predictions. This visualization also demonstrates LinearFold's improved prediction of long-distance base pairs than the baseline, as shown in Group I Mirabilis (bottom half, pair distance 250), 16S A. Pyrophilus (left part, 550), 23S E. coli (left part, 600). Fig. SI 3 shows the corresponding results comparing LinearFold-V with Vienna RNAfold. We also built a web demo visualizing results from all sequences in these three families.*

\section{Discussion}

RNA structure prediction is important for inferring RNA function and has many applications including drug design. The existing algorithms for RNA secondary structure prediction run in time that scales cubically with the sequence length, which is too slow for long non-coding RNAs; e.g., the baseline systems in this work, CONTRAfold and Vienna RNAfold, which are two of the most popular prediction software,

*http://web.engr.oregonstate.edu/ liukaib/demo_json+svg.html 
bioRxiv preprint doi: https://doi.org/10.1101/263509; this version posted February 14, 2018. The copyright holder for this preprint (which was not certified by peer review) is the author/funder, who has granted bioRxiv a license to display the preprint in perpetuity. It is made available under aCC-BY-NC-ND 4.0 International license.

take 2 hours and 1.7 hours, respectively, for a sequence of 32,753 nt. Furthermore, the existing algorithms also need memory space that scales quadratically with the sequence length, and as a result, both baseline systems fail to run on sequences beyond $32,767 \mathrm{nt}$. In reality, the longest RNA sequence in the RNAcentral dataset is $244,296 \mathrm{nt}$, which is $7 \times$ of that limit.

We design the first linear-time, linear-memory prediction algorithm, LinearFold, using dynamic programming plus beam search, and apply this algorithm to both machine-learned and thermodynamic models. The linearity in both time and memory is confirmed in Fig. 2. What is more interesting is the following three surprising findings:

1. Even though LinearFold uses only a fraction of runtime and memory compared to the existing algorithms in the baseline systems, our predicted structures are overall more accurate in both PPV and Sensitivity and on both machine-learned and thermodynamic models (see Fig. 3).

2. The accuracy improvement of LinearFold is more pronunced on longer families such as $16 \mathrm{~S}$ and $23 \mathrm{~S}$ rRNAs (see Figs. 3 and 5).

3. Even more surprisingly, LinearFold is also more accurate than the baselines on long-range base pairs that are more than 500 $n t$ apart (Fig. 4 E-F), which is well known to be a challenging problem for the current models (47).

4. Although the model depends on the beam size $b$, the number of base pairs and the accuracy of prediction are very robust to changes in beam size (when $b$ is in the range of 100-200).

Why our beam search algorithm, even though being approximate, outperforms the exact search baselines in terms of accuracy (esp. in $16 \mathrm{~S}$ and $23 \mathrm{~S}$ rRNAs)? First, current thermodynamic and machine learned models are far from perfect, so it is totally possible that a suboptimal structure (in terms of free energy or model score) is more accurate (in terms of PPV/Sensitivity) than the optimal structure. For example, for sequences of about 400 nucleotides, a structure about $80 \%$ correct can be found with a free energy within $5 \%$ of the optimal structure (52). But how does our algorithm systematically pick a more accurate suboptimal structure without seeing the ground truth? We suspect that it is because beam search prunes lower-scoring (sub)structures at each step, requiring the surviving (sub)structures to be highly scored at each prefix. This extra constraint might compensate for the inaccuracy of the model.

Our algorithm has several potential extensions. First of all, it might be possible to extend LinearFold to calculate the partition function and base pair probabilities, since the classical method for that task, the McCaskill algorithm (53), is similar to the cubic-time structure prediction algorithms which are used as baselines in this paper. Secondly, this linear-time algorithm to calculate base pair probabilities should facilitate the linear-time identification of pseudoknots, by replacing the cubic-time McCaskill algorithm with a linear-time one in those pseudoknot-prediction programs $(54,55)$. Thirdly, being linear-time, LinearFold also facilitate easier and faster training of parameters than the cubic-time CONTRAfold using structured prediction methods (56), and we envision a retrained model tailored to linear-time prediction should be even more accurate.

\section{Methods}

Our LinearFold approach is presented in four steps, starting from the most naive but easy-to-understand exhaustive search version (Fig. $6 \mathrm{~A}$ ), and gradually build it up to the linear-time version (Fig. 6 D), using a graph-structured stack and beam search.

The basic idea of linear-time prediction is to predict incrementally from left to right, labeling each nucleotide as unpaired ".", opening "(", or closing ")". We require this dot-bracket string to be well-balanced as we only consider pseudoknot-free structures.

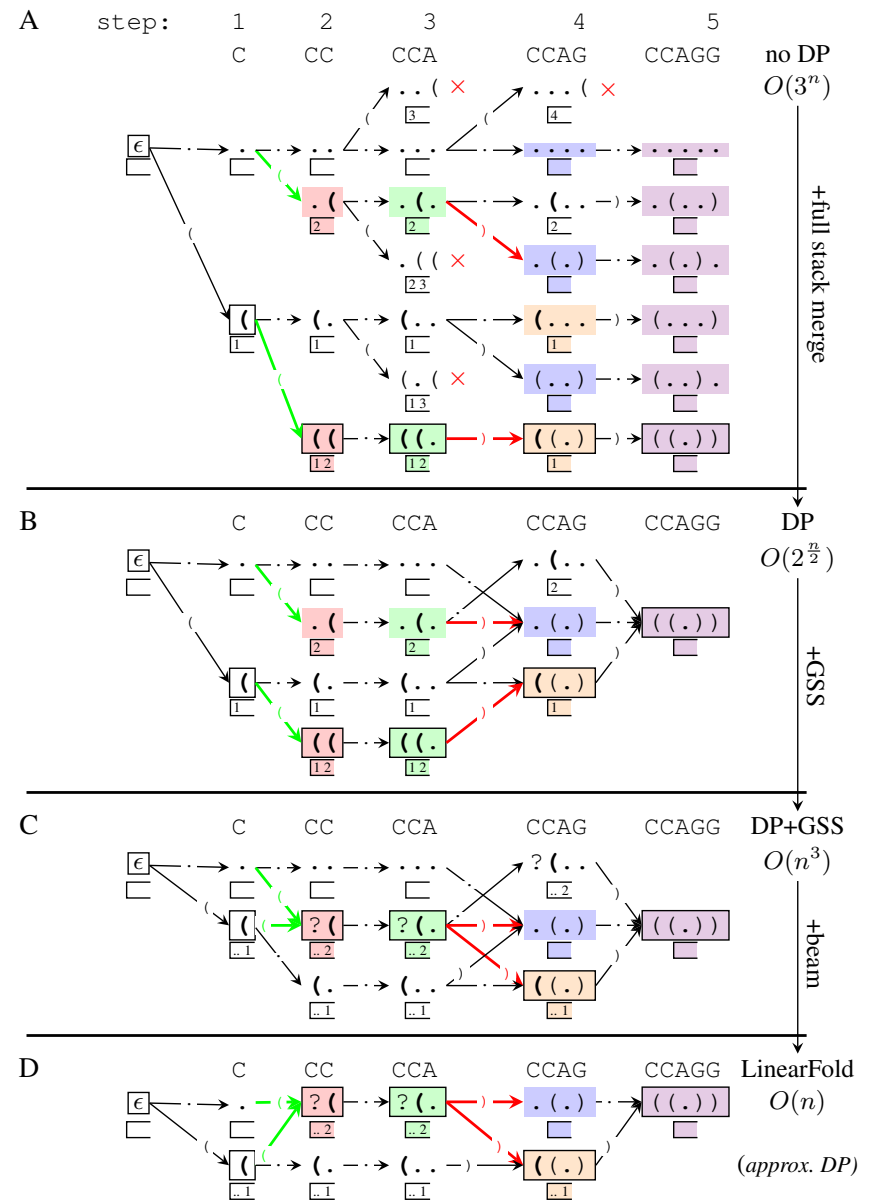

Fig. 6. Four-step demonstration of LinearFold, simply finding the max number of pairs instead of the actual MFE model. Each node represents a predicted prefix of the structure, with a stack showing the unpaired openings; each arrow corresponds to an action (push, skip, and pop); dead-end states are with a red $\times$. In C, nodes with the same color are merged by the Graph-Structured Stack. Nodes with borders represent the ground truth path.

Given an input RNA sequence $\mathbf{x}=x_{0} x_{1} \ldots x_{n-1}$ where $x_{i} \in\{\mathrm{A}, \mathrm{C}, \mathrm{G}, \mathrm{U}\}$, our algorithm aims to find the best structure $\mathbf{y}=y_{0} y_{1} \ldots y_{n-1}$ where $y_{i} \in$ $\{$ ".", "(", ")" $\}$ with minimum free energy (or minimum model cost):

$$
f(\mathbf{x})=\underset{\mathbf{y} \in \mathcal{Y}(\mathbf{x})}{\operatorname{argmin}} c(\mathbf{x}, \mathbf{y} ; \mathbf{w}) .
$$

Here $\mathcal{Y}(\mathbf{x})$ is the set of all possible structures, i.e., $\{\mathbf{y} \mid \mathbf{y}$ has balanced parentheses $\}, c$ is the cost function (i.e., free energy function), and $\mathbf{w}$ is the model (and parameters).

Naive exhaustive incremental prediction: $O\left(3^{n}\right)$ time. By exhaustively predicting $y$ from left-to-right, we traverse all the possible structures in $\mathcal{Y}(\mathbf{x})$, and pick the one with the minimum free energy or model cost. We formalize each state at step $j$ ( $j \in$ $\{0, \ldots, n\})$ to be a triple, $s=\langle\sigma \mid i, j\rangle: \mathbf{y}$, where $\sigma \mid i$ is a stack consisting of unmatched openings so far where $i$ is the top of the stack, meaning $x_{i}$ is the last unmatched opening nucleotide. $\mathbf{y}$ is the corresponding dot-bracket (sub)sequence up to $x_{j-1}$. For each state, it can transition to a subsequent state, taking one of the three actions: push, which labels the current nucleotide $x_{j}$ as a left bracket "(", putting it on top of the stack, skip, which labels $x_{j}$ as a dot ".", leaving the stack unchanged, and pop, which labels $x_{j}$ as a right bracket ")", if it matches $x_{i}$ and popping $i$ from the stack. See Fig. SI 4 (a) for the deductive system. This algorithm takes $O\left(3^{n}\right)$ time to exhaustively traverse all possible structures (see Figure 6 A).

Dynamic Programming via Full Stack Merging: $O\left(2^{\frac{n}{2}}\right)$ time. Now we apply dynamic programming on top of this exhaustive method to exploit shared computations. Consider a simple case that two states can be merged: if there are two states in the same step $j,\langle\sigma, j\rangle: \mathbf{y}$ and $\langle\sigma, j\rangle: \mathbf{y}^{\prime}$, sharing the exact same stack $\sigma$ but with different dot-bracket strings $\mathbf{y}$ and $\mathbf{y}^{\prime}$, we say that these two states are "equivalent" and we can merge them (and only keep the better scoring between $\mathbf{y}$ and $\mathbf{y}^{\prime}$ ). Fig. $6 \mathrm{~B}$ illustrates this merging. Although we merge to reduce the number of states, it is still exponential time, 
bioRxiv preprint doi: https://doi.org/10.1101/263509; this version posted February 14, 2018. The copyright holder for this preprint (which was not certified by peer review) is the author/funder, who has granted bioRxiv a license to display the preprint in perpetuity. It is made available under aCC-BY-NC-ND 4.0 International license.

since there could be exponentially many different stacks in each step. This algorithm takes $O\left(2^{\frac{n}{2}}\right)$ time.

Dynamic Programming via Graph Structured Stacks: $O\left(n^{3}\right)$ time. To avoid considering exponentially many states, we further merge states with different stacks. Consider two states in the same step $j,\left\langle\sigma_{0} \mid i, j\right\rangle$ and $\left\langle\sigma_{1} \mid i, j\right\rangle$, which share the last unpaired opening $i$ (i.e., stack top). We call these states "temporarily equivalent", since they can be treated as exactly the same until the unpaired opening $x_{i}$ is closed (and thus popped from the stack). In other words we can represent both stacks $\sigma_{0} \mid i$ and $\sigma_{1} \mid i$ as ...i where ... denotes part of the history that we do not care at this moment. This factorization of stacks is called "Graph-Structured Stacks" (GSS) by Tomita (46). After merging, we define the new state to be $\langle i, j\rangle$ and therefore we maintain $O\left(n^{2}\right)$ states. For each state $\langle i, j\rangle$, the pop action can take worst-case $O(n)$ time because $\langle i, j\rangle$ can combine with every $\langle k, i\rangle$ from step $i$. Thus the overall time complexity is $O\left(n^{3}\right)$. See Fig. $6 \mathrm{C}$ for an example of the merging process and Fig. SI 4 (b) for the deductive system.

Dynamic Programming via Beam Search: $O(n)$ time. In practice, the exact search algorithm still runs in $O\left(n^{3}\right)$ time. But this left-to-right $O\left(n^{3}\right)$ search is easily "linearizable" unlike the traditional bottom-up $O\left(n^{3}\right)$ search used by all existing systems for RNA structure prediction. We further employ beam search pruning (56) to reduce the complexity to linear time. Generally, we only keep the $b$ top-scoring states $\langle i, j\rangle$ for each step. This way all the lower-scoring states are pruned, and if a structure survives to the end, it must have been one of the top $b$ states in every step. This pruning also means that in a pop action, a state $(i, j)$ can combine with at most $b$ states $(k, i)$ from step $i$ Thus the overall time complexity is $O\left(n b^{2}\right)$. However, instead of generating $b^{2}$ new states from a pop action, we use cube pruning (57) to generate the best $b$ states, which would take $O(b \log b)$ time. Thus the overall running time over a length- $n$ sequence is $O(n b \log b)$, see See Figure $6 \mathrm{D}$ for beam search.

Dataset, Evaluation Metrics and Significance Testing. We choose the ArchiveII dataset (48), a diverse set of over 3,000 RNA sequences with known secondary structures. But since the current CONTRAfold machine-learned model (v2.02) is trained on the S-Processed dataset (58) we removed those sequences appeared in the S-Processed dataset. The resulting dataset we used contains 2,889 sequences over 9 families, with an average length of $222.2 \mathrm{nt}$. Due to the uncertainty of base-pair matches existing in comparative analysis, we consider a base pair to be correctly predicted if it is also slipped by one nucleotide on a strand, accordingly $((48))$. Generally, if a pair $(i, j)$ is in the predicted structure, we claim it's correct if one of $(i, j),(i-1, j),(i+1, j),(i, j-1)$, $(i, j+1)$ is in the ground truth structure. We report both Sensitivity and PPV where

Sensitivity $=\frac{\text { true positives }}{\text { true positives }+ \text { false negatives }}, \mathrm{PPV}=\frac{\text { true positives }}{\text { true positives }+ \text { false positives }}$

We use the paired two-tailed $t$-test to calculate the statistical significance, with the type I error rate, consistent with the previous methods (51).

ACKNOWLEDGMENTS. This work was partially supported by NSF grant 1656051 (L.H.), NIH grants R56 AG053460 and R21 AG052950 (D.H.), and NIH grant R01GM076485 (D.H.M.). We thank James Cross for algorithm design, Kaibo Liu for the web demo, and Juneki Hong for proofreading.

1. Eddy SR (2001) Non-coding RNA genes and the modern RNA world. Nature Reviews Genetics 2(12):919-929.

2. Doudna JA, Cech TR (2002) The chemical repertoire of natural ribozymes. Nature 418(6894):222-228.

3. Scott WG (2007) Ribozymes. Current opinion in structural biology 17(3):280-286.

4. Storz G, Gottesman S (2006) 20 versatile roles of small RNA regulators in bacteria. Cold Spring Harbor Monograph Archive 43:567-594.

5. Wu L, Belasco JG (2008) Let me count the ways: mechanisms of gene regulation by miRNAs and siRNAs. Molecular cell 29(1):1-7.

6. Serganov A, Nudler E (2013) A decade of riboswitches. Cell 152(1):17-24.

7. Bachellerie JP, Cavaillé J, Hüttenhofer A (2002) The expanding snoRNA world. Biochimie 84(8):775-790.

8. Wahl MC, Will CL, Lührmann R (2009) The spliceosome: design principles of a dynamic rnp machine. Cell 136(4):701-718.

9. Walter $\mathrm{P}$, Blobel G (1982) Signal recognition particle contains a 7s RNA essential for protein Nature 299:21

10. Johnsson P, Lipovich L, Grandér D, Morris KV (2014) Evolutionary conservation of long noncoding RNAs; sequence, structure, function. Biochimica et Biophysica Acta (BBA)-General Subjects 1840(3):1063-1071.

11. Gilbert W (1986) Origin of life: The RNA world. Nature 319(6055).

12. Joyce GF (1994) In vitro evolution of nucleic acids. Current opinion in structural biology 4(3):331-336.

13. Sazani P, et al. (2002) Systemically delivered antisense oligomers upregulate gene expression in mouse tissues. Nature biotechnology 20(12):1228-1233.

14. Crooke ST (2004) Antisense strategies. Current molecular medicine 4(5):465-487.

15. Childs-Disney JL, Wu M, Pushechnikov A, Aminova O, Disney MD (2007) A small molecule microarray platform to select RNA internal loop- ligand interactions. ACS chemical biology 2(11):745-754

16. Gareiss PC, et al. (2008) Dynamic combinatorial selection of molecules capable of inhibiting the (cug) repeat RNA- mbnl1 interaction in vitro: discovery of lead compounds targeting myotonic dystrophy (dm1). Journal of the American Chemical Society 130(48):16254-16261.

17. Castanotto D, Rossi JJ (2009) The promises and pitfalls of RNA-interference-based therapeutics. Nature 457(7228):426-433.
18. Palde PB, Ofori LO, Gareiss PC, Lerea J, Miller BL (2010) Strategies for recognition of stemloop RNA structures by synthetic ligands: Application to the hiv-1 frameshift stimulatory sequence. Journal of medicinal chemistry 53(16):6018-6027.

19. Seetin MG, Mathews DH (2012) RNA structure prediction: an overview of methods. Bacterial Regulatory RNA: Methods and Protocols pp. 99-122.

20. Hofacker IL, Lorenz R (2014) Predicting RNA structure: advances and limitations. RNA Folding: Methods and Protocols pp. 1-19.

21. Gruber A, Findeiss S, Washietl S, Hofacker I, Stadler PF (2010) RNAz 2.0: improved noncoding RNA detection in Pacific Symposium on Biocomputing. Vol. 15, pp. 69-79.

22. Washietl S, et al. (2012) Computational analysis of noncoding RNAs. Wiley Interdisciplinary Reviews: RNA 3(6):759-778.

23. Fu Y, Xu ZZ, Lu ZJ, Zhao S, Mathews DH (2015) Discovery of novel ncRNA sequences in multiple genome alignments on the basis of conserved and stable secondary structures. PloS one 10(6):e0130200.

24. Lu ZJ, Mathews DH (2008) Efficient siRNA selection using hybridization thermodynamics. Nucleic acids research 36(2):640-647.

25. Tafer $\mathrm{H}$, et al. (2008) The impact of target site accessibility on the design of effective sirnas. Nature biotechnology 26(5):578-583.

26. Stephens ZD, et al. (2015) Big data: astronomical or genomical? PLoS Biol 13(7):e1002195

27. Sloma MF, Mathews DH (2015) Chapter four-improving RNA secondary structure prediction with structure mapping data. Methods in enzymology 553:91-114.

28. Ding $Y$, et al. (2014) In vivo genome-wide profiling of RNA secondary structure reveals novel regulatory features. Nature 505(7485):696-700.

29. Flynn RA, et al. (2016) Transcriptome-wide interrogation of RNA secondary structure in living cells with icshape. Nature protocols 11(2):273-290.

30. Rouskin S, Zubradt M, Washietl S, Kellis M, Weissman JS (2014) Genome-wide probing of RNA structure reveals active unfolding of mrna structures in vivo. Nature 505(7485):701-705.

31. Ouyang Z, Snyder MP, Chang HY (2013) Seqfold: genome-scale reconstruction of RNA secondary structure integrating high-throughput sequencing data. Genome research 23(2):377387.

32. Spasic A, Assmann SM, Bevilacqua PC, Mathews DH (2017) Modeling RNA secondary structure folding ensembles using shape mapping data. Nucleic acids research.

33. Wu Y, et al. (2015) Improved prediction of RNA secondary structure by integrating the free energy model with restraints derived from experimental probing data. Nucleic acids research 43(15):7247-7259.

34. Cheng CY, Kladwang W, Yesselman JD, Das R (2017) RNA structure inference through chemical mapping after accidental or intentional mutations. Proceedings of the National Academy of Sciences p. 201619897.

35. Tian S, Das R (2016) RNA structure through multidimensional chemical mapping. Quarterly reviews of biophysics 49 .

36. Mathews DH, Turner DH (2006) Prediction of RNA secondary structure by free energy minimization. Current opinion in structural biology 16(3):270-278.

37. Lorenz R, et al. (2011) ViennaRNA package 2.0. Algorithms for Molecular Biology 6(1):1

38. Do CB, Woods DA, Batzoglou S (2006) Contrafold: RNA secondary structure prediction with out physics-based models. Bioinformatics 22(14):e90-e98.

39. Rivas E, Lang R, Eddy SR (2012) A range of complex probabilistic models for RNA secondary structure prediction that includes the nearest-neighbor model and more. RNA 18(2):193-212.

40. Zuker M, Stiegler $P$ (1981) Optimal computer folding of large RNA sequences using thermodynamics and auxiliary information. Nucleic acids research 9(1):133-148.

41. Waterman MS, Smith TF (1986) Rapid dynamic programming algorithms for rna secondary structure. Advances in Applied Mathematics 7(4):455-464

42. Kasami T (1965) An efficient recognition and syntax analysis algorithm for context-free languages, (Air Force Cambridge Research Lab), Technical Report AFCRL-65-758.

43. Younger $\mathrm{DH}$ (1967) Recognition and parsing of context-free languages in time $n^{3}$. Information and Control 10:189-208.

44. Lange SJ, et al. (2012) Global or local? predicting secondary structure and accessibility in mrnas. Nucleic acids research 40(12):5215-5226.

45. Huang L, Sagae K (2010) Dynamic programming for linear-time incremental parsing in Proceedings of ACL 2010. (Uppsala, Sweden).

46. Tomita M (1988) Graph-structured stack and natural language parsing in Proc. ACL.

47. Amman F, et al. (2013) The trouble with long-range base pairs in RNA folding in Brazilian Symposium on Bioinformatics. (Springer), pp. 1-11.

48. Sloma M, Mathews D (2016) Exact calculation of loop formation probability identifies folding motifs in RNA secondary structures. RNA, In Press.

49. RNAcentral Consortium, , et al. (2017) RNAcentral: a comprehensive database of non-coding RNA sequences. Nucleic acids research 45(D1):D128-D134.

50. Zhao Y, et al. (2016) Noncode 2016: an informative and valuable data source of long noncoding rnas. Nucleic acids research 44(D1):D203-D208.

51. Xu Z, Almudevar A, Mathews DH (2011) Statistical evaluation of improvement in RNA secondary structure prediction. Nucleic acids research 40(4):e26-e26.

52. Zuker M, Jaeger JA, Turner DH (1991) A comparison of optimal and suboptimal rna secondary structures predicted by free energy minimization with structures determined by phylogenetic comparison. Nucleic acids research 19(10):2707-2714.

53. McCaskill JS (1990) The equilibrium partition function and base pair binding probabilities for RNA secondary structure. Biopolymers 29(6-7):1105-1119.

54. Bellaousov S, Mathews DH (2010) Probknot: fast prediction of RNA secondary structure including pseudoknots. Rna 16(10):1870-1880.

55. Sato K, Kato Y, Hamada M, Akutsu T, Asai K (2011) Ipknot: fast and accurate prediction of RNA secondary structures with pseudoknots using integer programming. Bioinformatics 27(13):i85-i93.

56. Huang L, Fayong S, Guo Y (2012) Structured perceptron with inexact search in Proc. NAACL.

57. Huang L, Chiang D (2007) Forest rescoring: Fast decoding with integrated language models in Proceedings of ACL 2007.

58. Andronescu M, Condon A, Hoos H, Mathews D, Murphy K (2007) Efficient parameter estimation for RNA secondary structure prediction. Bioinformatics, ISMB/ECCB 2007. 


\section{Supporting Information}

\section{LinearFold: Linear-Time Prediction of RNA Secondary Structures}

Dezhong Deng, Kai Zhao, David Hendrix, David H. Mathews, and Liang Huang 
bioRxiv preprint doi: https://doi.org/10.1101/263509; this version posted February 14,2018 . The copyright holder for this preprint (which was not certified by peer review) is the author/funder, who has granted bioRxiv a license to display the preprint in perpetuity. It is made available under aCC-BY-NC-ND 4.0 International license.

\begin{tabular}{|c|c|c|c|c|c|c|c|c|c|c|c|}
\hline \multirow[b]{2}{*}{ Family } & \multicolumn{2}{|c|}{ \# of sequences } & \multirow{2}{*}{$\begin{array}{l}\text { avg. } \\
\text { length }\end{array}$} & \multicolumn{2}{|c|}{ CONTRAfold MFE } & \multicolumn{2}{|c|}{ LinearFold-C $b=100$} & \multicolumn{2}{|c|}{ Vienna RNAfold } & \multicolumn{2}{|c|}{ LinearFold- $V b=100$} \\
\hline & total & used & & PPV & Sensitivity & $\triangle P P V$ & $\Delta$ Sensitivity & PPV & Sensitivity & $\triangle \mathrm{PPV}$ & $\Delta$ Sensitivity \\
\hline tRNA & 557 & 74 & 77.3 & 69.32 & 70.84 & +0.00 & +0.00 & 63.80 & 73.25 & -0.13 & +0.00 \\
\hline 5S rRNA & 1,283 & 1,125 & 118.8 & 76.48 & 76.62 & +0.13 & +0.01 & 60.12 & 66.62 & +0.01 & +0.04 \\
\hline SRP & 928 & 886 & 186.1 & 64.24 & 63.88 & -0.03 & -0.03 & 60.93 & 66.66 & $\left(^{*}\right)+0.38$ & $\left(^{*}\right)+0.29$ \\
\hline RNaseP & 454 & 182 & 344.1 & 49.34 & 48.10 & -0.15 & -0.17 & 47.78 & 55.79 & +0.03 & -0.19 \\
\hline tmRNA & 462 & 462 & 366.0 & 46.50 & 39.70 & $\left(^{*}\right)-0.62$ & $\left(^{*}\right)-0.76$ & 42.09 & 47.58 & $\left({ }^{*}\right)-0.96$ & $\left(^{*}\right)-1.00$ \\
\hline Group I Intron & 98 & 96 & 424.9 & 52.50 & 50.78 & $\left({ }^{* *}\right)+1.30$ & $(*)+0.94$ & 47.02 & 57.96 & $\left(^{*}\right)+0.80$ & $\left({ }^{*}\right)+0.94$ \\
\hline lomerase RNA & 37 & 37 & 444.6 & 46.12 & 59.96 & -0.07 & -0.05 & 41.87 & 58.77 & -0.01 & -0.14 \\
\hline 16S rRNA & 22 & 22 & $1,547.9$ & 40.46 & 41.48 & $\left({ }^{*}\right)+3.89$ & $\left({ }^{*}\right)+3.08$ & 37.63 & 44.60 & $\left(^{*}\right)+1.46$ & $\left(^{*}\right)+1.56$ \\
\hline $23 \mathrm{~S}$ rRNA & 5 & 5 & $2,927.4$ & 48.29 & 49.85 & $\left({ }^{* *}\right)+14.00$ & $\left({ }^{* *}\right)+9.98$ & 55.09 & 62.67 & +0.40 & +0.25 \\
\hline Overall & 3,846 & 2,889 & 222.2 & 54.80 & 55.69 & +2.06 & +1.44 & 50.70 & 59.32 & +0.22 & +0.20 \\
\hline
\end{tabular}

Table SI 1. Detailed information of the Archivell dataset and the prediction accuracies of CONTRAfold MFE, LinearFold-C, Vienna RNAfold and LinearFold- $V$. Statistical significance are marked by ${ }^{\star}(0.01 \leq p<0.05)$ and ${ }^{\star *}(p<0.01)$.
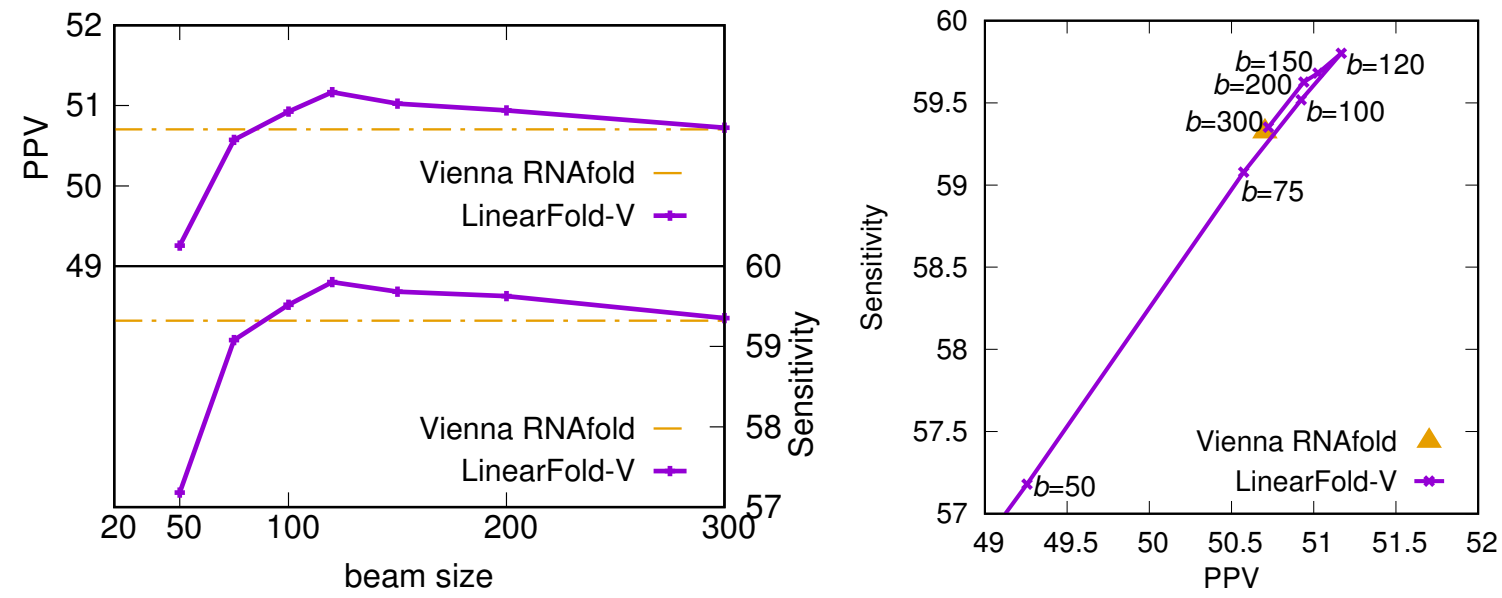

Fig. SI 1. This figure corresponds to Figure 4(c)(d) but with the ViennaRNA version, running on the Archivell dataset. Left: trend of both PPV and Sensitivity with the increasing of beam size; right: PPV and Sensitivity of LinearFold by beam size.
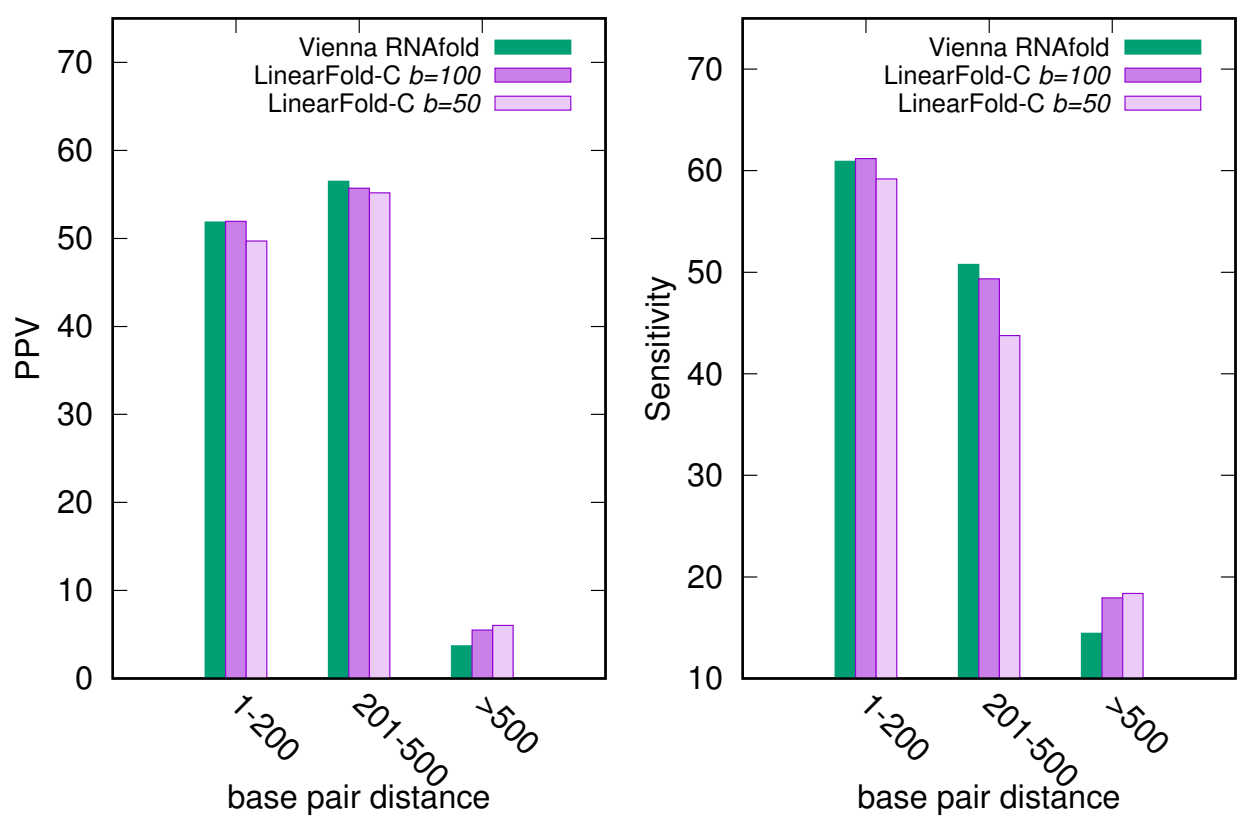

Fig. SI 2. This figure corresponds to Figure4(c)(d), with the ViennaRNA version, running on the Archivell dataset. It shows PPV and Sensitivity of LinearFold-V by pair length in the Mathews dataset, comparing to Vienna RNAfold. Each bar represents the overall PPV/Sensitivity of all the base pairs in a length range. 
bioRxiv preprint doi: https://doi.org/10.1101/263509; this version posted February 14,2018 . The copyright holder for this preprint (which was not certified by peer review) is the author/funder, who has granted bioRxiv a license to display the preprint in perpetuity. It is made available under aCC-BY-NC-ND 4.0 International license.

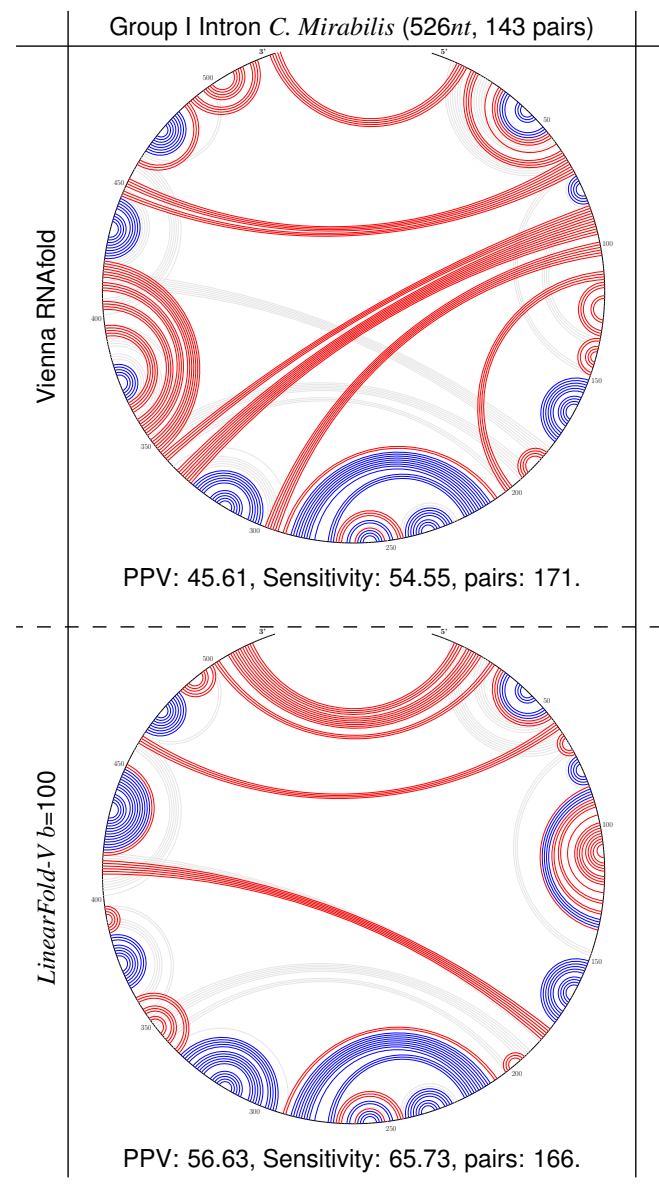

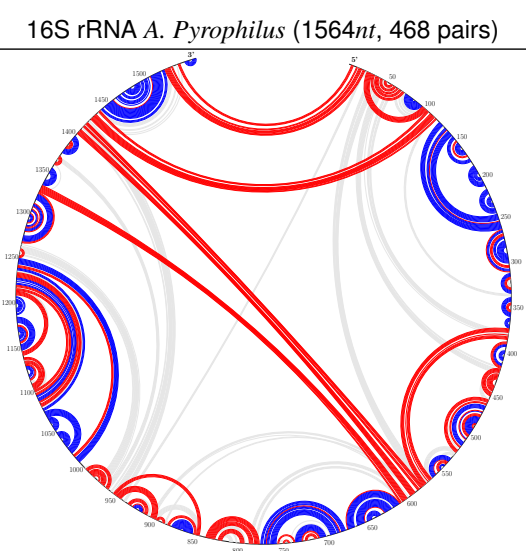

PPV: 56.12, Sensitivity: 63.68, pairs: 531.

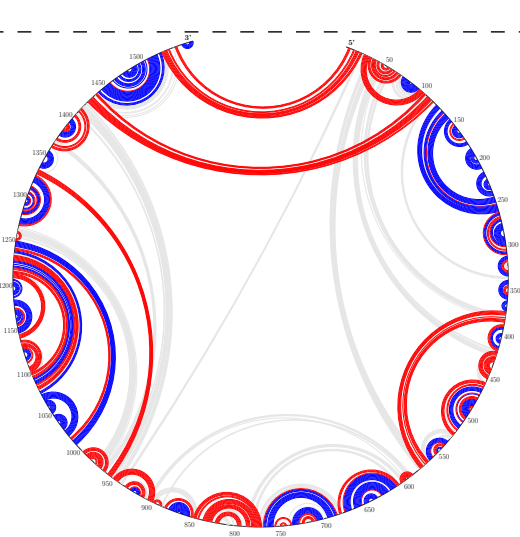

PPV: 55.81, Sensitivity: 62.61, pairs: 525.

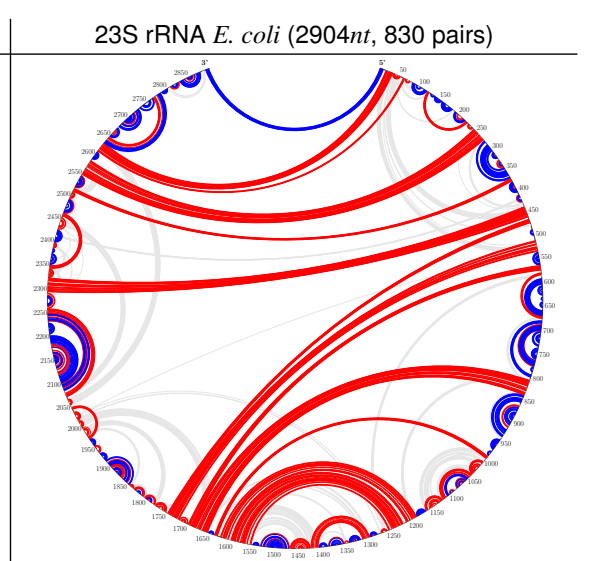

PPV: 52.34, Sensitivity: 57.83, pairs: 917.

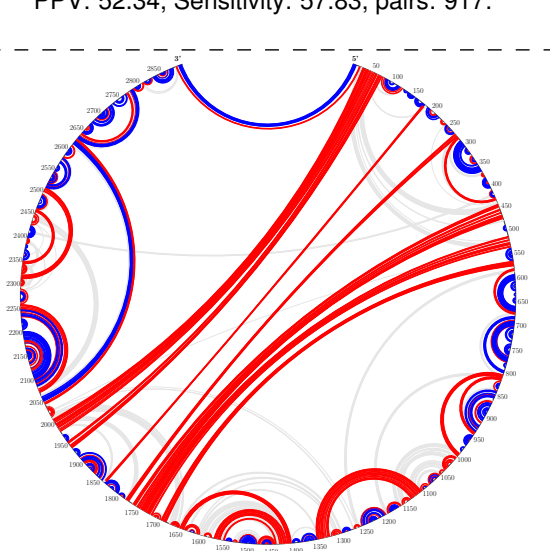

PPV: 56.14 , Sensitivity: 62.44, pairs: 928.

Fig. SI 3. Circular plots of 3 RNA sequences (corresponding to Figure 5) comparing Vienna RNAfold with LinearFold-V.

$$
\begin{aligned}
& \text { input } \quad x_{0} \ldots x_{n-1} \\
& x_{0} \ldots x_{n-1} \\
& \text { state }\langle\sigma, j\rangle: \mathbf{y} \quad\langle i, j\rangle: \mathbf{y} \\
& \text { axiom }\left\langle\sigma_{\epsilon}, 0\right\rangle: \epsilon \quad\langle-1,0\rangle: \epsilon \\
& \text { goal }\left\langle\sigma_{\epsilon}, n\right\rangle: \mathbf{y} \quad\langle-1, n\rangle: \mathbf{y} \\
& \text { push } \frac{\langle\sigma, j\rangle: \mathbf{y}}{\langle\sigma \mid j, j+1\rangle: \mathbf{y}{ }^{\prime \prime}(’} \quad \frac{\langle i, j\rangle: \mathbf{y}}{\langle j, j+1\rangle: \mathbf{y}{ }^{\prime \prime}('} \\
& \operatorname{skip} \frac{\langle\sigma, j\rangle: \mathbf{y}}{\langle\sigma, j+1\rangle: \mathbf{y}{ }^{\prime} . '} \quad \frac{\langle i, j\rangle: \mathbf{y}}{\langle i, j+1\rangle: \mathbf{y} \circ^{\prime},} \\
& \text { pop } \frac{\langle\sigma \mid i, j\rangle: \mathbf{y}}{\left.\langle\sigma, j+1\rangle: \mathbf{y}^{\prime}\right)^{\prime}} \text { if }\left(x_{i}, x_{j}\right) \text { match } \frac{\langle k, i\rangle: \mathbf{y}^{\prime}\langle i, j\rangle: \mathbf{y}}{\left.\langle k, j+1\rangle: \mathbf{y}^{\prime} \circ \mathbf{y} \circ^{\prime}\right)^{\prime}} \text { if }\left(x_{i}, x_{j}\right) \text { match } \\
& \begin{array}{ll}
\text { (a) exhaustive, } O\left(3^{n}\right) & \text { (b) DP with GSS, } O\left(n^{3}\right)
\end{array}
\end{aligned}
$$

Fig. SI 4. Deductive system comparing the exhaustive search algorithm and Dynamic Programming with Graph Structured Stack. Here o denotes string concatenation. We say $(a, b)$ "matches" if $(a, b)$ is one of the allowed pairs (C-G, A-U, G-U). 